\title{
Non-Resolving Lung Infiltrate: A Rare Case of Papillary Adenocarcinoma of the Lung
}

\author{
Nikhil Madan ${ }^{1}$, Carolina Pinzon Escobar ${ }^{2}$ and Vipul Patel ${ }^{1, *}$ \\ ${ }^{1}$ Division of Pulmonary and Critical Care Medicine, Newark Beth Israel Medical Center, RWJ Barnabas Health, Newark New Jersey, USA \\ ${ }^{2}$ Department of Medicine Newark Beth Israel Medical Center, RWJ Barnabas Health, Newark, New Jersey, USA
}

${ }^{*}$ Corresponding author: Vipul Patel, MD, Associate Medical Director of Lung Transplant, Division of Pulmonary \& Critical Care, New Jersey, USA, Tel: +19735241839, E-mail: vipul.patel@rwjbh.org

Received date: November 12, 2019; Accepted date: December 09, 2019; Published date: December 16, 2019

Citation: Madan N, Patel V, Escobar CP (2019) Non-Resolving Lung Infiltrate: A Rare Case of Papillary Adenocarcinoma of the Lung. Med Case Rep Vol.5 No.3:127.

\section{Abstract}

Lung carcinoma is one of most common and leading cause of cancer related mortality in United States. The main sub-types of lung cancer are non-small cell lung carcinoma (NSCLC) and small cell carcinoma (SCLC). About $85 \%$ of lung cancers are of the non-small cell type, of which more than $50 \%$ are adenocarcinomas. Primary papillary adenocarcinoma (PA) is a subtype of adenocarcinoma with predominance of papillary structures that replace the underlying alveolar architecture supported by fibro-vascular cores with complicated secondary and tertiary branches. PA is a relatively rare type that can present a diagnostic challenge when it presents as a non-resolving infilitrate as seen in this case. It is more common in woman and non-smokers. The subtype of adenocarcinoma is key factor for therapeutic choice of chemotherapy. The case reviewed here is a rare presentation of a middle aged chronic smoker Man with past medical history of chronic obstructive pulmonary disease (COPD) who was treated several times for recurrent episodes of worsening dyspnea attributed to an infectious etiology, but without any significant improvement which led to further testing and trans-bronchial biopsy which reveled PA of the lung. Tissue diagnosis is therefore warranted in patients with a non-resolving lung infiltrate.

Keywords: Lung carcinoma; Cancer; Primary papillary adenocarcinoma; Chronic Obstructive Pulmonary Disease (COPD)

\section{Introduction}

A non-resolving infilitrate is encountered in clinical practice not uncommonly. It does present a diagnostic dilemma for the clinician as the differential diagnosis is vast. Lung cancer specially adenocarcinoma is known to present as a nonresolving infiltrate. Further evaluation with special studies and tissue biopsy as indicated should be pursued in such cases. Lung carcinoma is the one of the most common cancer worldwide and its incidence is gradually increasing. It is a leading cause of cancer related death in United States. About $85 \%$ of all lung cancer is Non-small cell lung cancer (NSCLC) and adenocarcinoma account for more than $50 \%$ of these cases [1]. Primary papillary adenocarcinoma (PA) is an uncommon invasive form of lung cancer where papillae replace the underlying alveolar architecture. In 1997, Silver and Askins described a series of primary lung neoplasms, which they classified on the basis of histologic features. In this series, PA was diagnosed when more than $75 \%$ of neoplasm contained papillary architecture supported by fibro-vascular cores with complicated secondary and tertiary branches [2]. The WHO classification defines PA as adenocarcinoma with predominance of papillary structures that replace the underlying alveolar architecture [3]. PA is distinct clinicpathologic entity with considerably worse morbidity and mortality compared to other sub-types of adenocarcinoma [4]. Radiologically, PA presents as solitary nodule, consolidation or multi-centric, diffuse disease [5]. We would like to report a case of PA presented with non-specific radiologic findings. Patient was treated extensively for pulmonary infections empirically without appropriate clinical response.

\section{Case Report}

This is a 62-year-old man who was referred to our institute for further evaluation of a non-resolving lung infiltrate. He initially presented to the outside hospital with episodic dyspnea on exertion and cough with small amount of yellow sputum production for about two months duration. He did not report fever, malaise or lethargy associated with cough. He had significant weight loss of about 20 pounds in last three months. Patient received treatment with cortico-steroids and few courses of oral anti-bacterial therapy for persistent symptoms and non-resolving lung infiltrate. After every course of antibiotics, he had partial relief of symptoms; however this was short-lasting. Patient was noted to become progressively hypoxic requiring supplemental oxygen via nasal cannula at 5 Liters per minute (LPM) He had undergone bronchoscopy with broncho-alveolar lavage at the outside facility, which did not reveal any specific organism or lead to any diagnosis. 
Past medical history includes chronic hepatitis $C$ infection treated with Ledipasvir and Sofosbuvir combination, cirrhosis of liver secondary to chronic hepatitis $C$ infection, chronic obstructive pulmonary disease (COPD) likely due to long history of cigarette smoking (57 pack years). He had poly substance abuse in past includes alcohol, marijuana and intravenous opiates, requiring rehabilitation with methadone.

Patient was referred to our facility for further management. On arrival to our hospital, patient was noted to have mild respiratory distress. He had significant cachexia and muscle wasting. His blood pressure and heart rate were within normal limits. His oxygen saturations were at $91 \%$ on oxygen supplementation at 5 LPM.

There were decreased breath sounds on right lower part of lung with few rhonchi. Rest of physical examination was within normal limits. Arterial blood gas showed partial pressure of oxygen was $55 \mathrm{~mm} \mathrm{Hg}$. Chest radiograph showed increased lung size and flattened diaphragms bilaterally, consistent with COPD. There was right middle and lower lobe opacity suggestive of pneumonia (Figure 1).

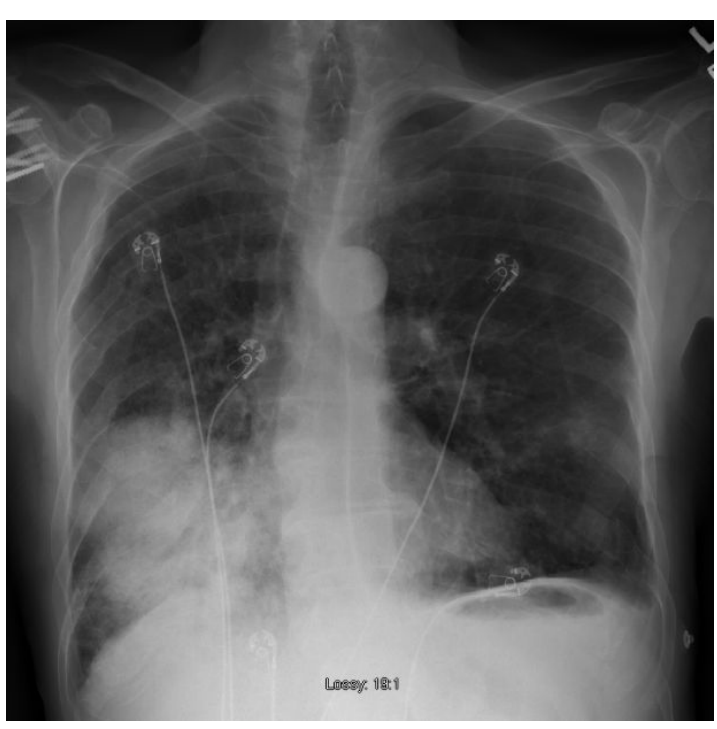

Figure 1: Chest $\mathrm{x}$-ray showed right middle and lower lobe opacity suggestive of pneumonia.

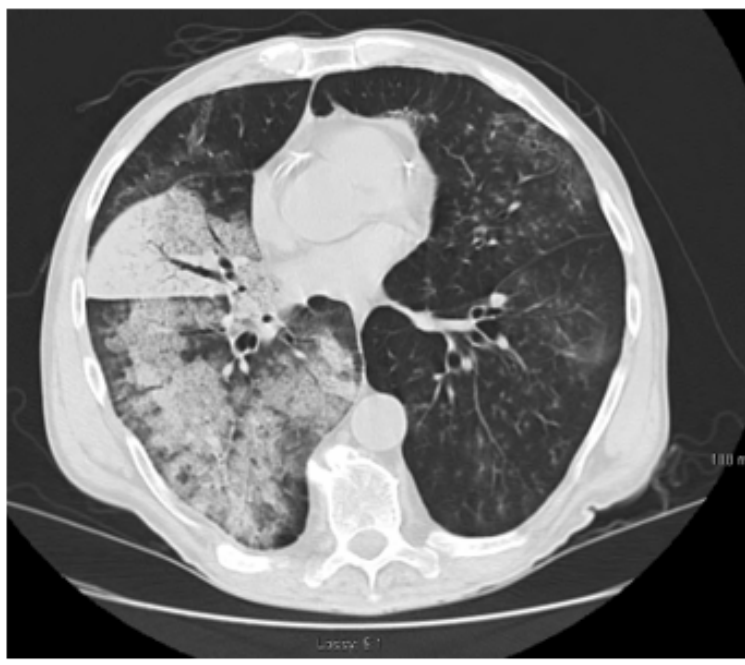

(A)

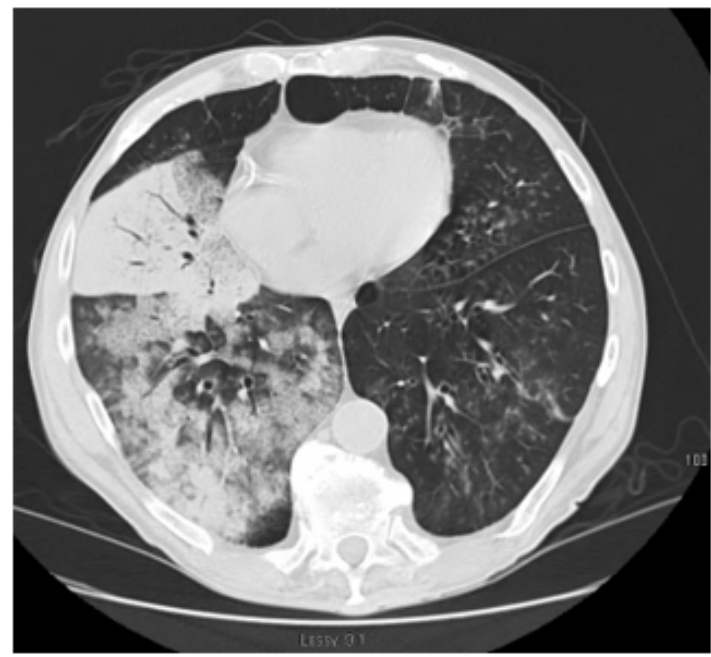

(B)

Figure 2: (A and B) CT scan of chest showed air bronchogram along with ground glass opacity in right middle and lower lobes.

Chest computed tomography (CT) scan showed right middle lobe opacity with air bronchograms. Confluent opacity with air bronchogram along with ground glass opacity was noted on right lower lobe (Figure 2). There was no evidence of lymph node enlargement in hilar or mediastinal region. Thyroid gland appeared normal on CT scan of chest. Spirometry showed moderate reduction in forced expiratory volume in one second (FEV1) and forced vital capacity (FVC) with significant obstructive pattern. He had severe reduction in diffusion capacity noted on study performed using carbon monoxide.

Differential diagnosis included but not limited to indolent bacterial infection, fungal pneumonia, Mycobacterium infection, interstitial lung disease, pulmonary vasculitis and malignancy. Patient underwent trans-bronchial biopsy of the lung to further investigate this. Biopsy revealed majority of the tissue obtained displaying papillary architecture (Figure 3).

The papillae lined by round to oval cells with hyper chromatic nuclei. These findings were consistent with papillary adenocarcinoma. Immuno-histochemical staining with thyroid transcription factor - 1 (TTF-1) was positive suggestive of primary lung cancer (Figure 4).

Programmed death ligand 1 (PD-L1) immuno-histochemical analysis showed tumor proportion score was 2-3\% (low positive). Fluorescent in situ hybridization (FISH) showed no evidence of epidermal growth factor receptor (EGFR), anaplastic lymphoma kinase (ALK) and Proto-oncogene 
tyrosine-protein kinase ROS (ROS1) gene rearrangement. KRAS p.Gly12Val genomic alteration was detected.

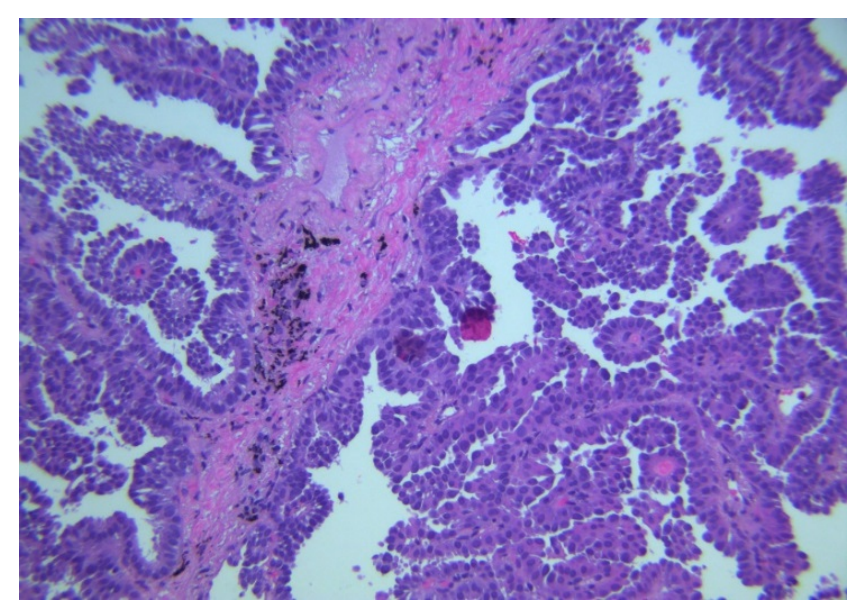

Figure 3: Transbronchial biopsy of lung revealed majority of the tissue obtained displaying papillary architecture.

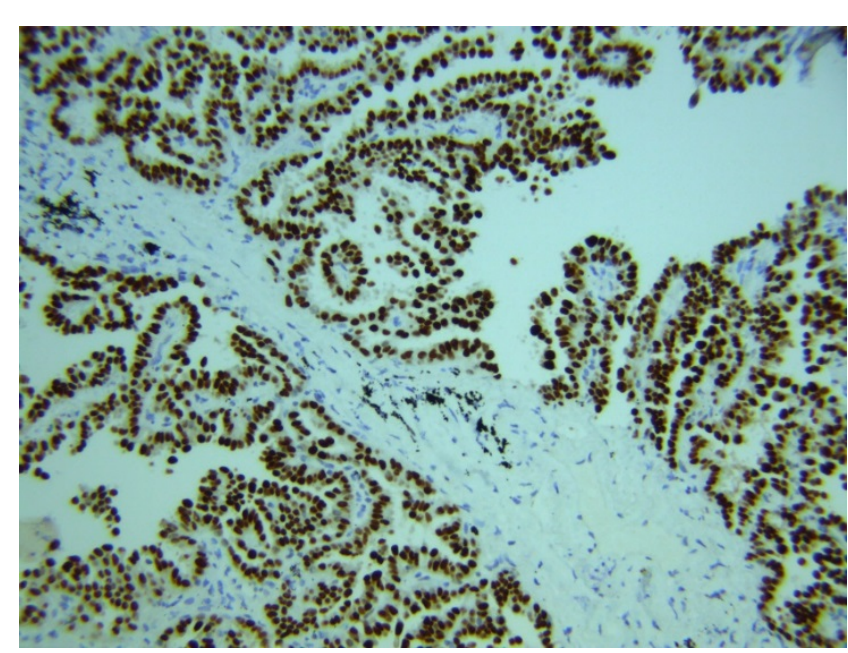

Figure 4: Immuno-histochemical staining with thyroid transcription factor - 1 (TTF-1) was positive suggestive of primary lung cancer.

Further investigation with bone scan and CT scan of chest abdomen and pelvis with intravenous contrast showed no evidence of disease outside of chest.

These findings were discussed in a multidisciplinary team including a pulmonologist, oncologist, radiation oncologist, radiologist, pathologist and thoracic surgeon revealed that patient was not a surgical candidate due to poor underlying lung function. He was not a candidate for systemic chemotherapy or immunotherapy due to poor functional status. After extensive discussion with patient, he decided to go with palliative care and pursued comfort measures.

\section{Discussion}

Lung cancer is the leading cause of cancer deaths in the world. Adenocarcinoma is the most common type of lung cancer. Recent International Association for the Study of Lung Cancer (IASLC)/the American Thoracic Society (ATS)/the European Respiratory Society (ERS) pathologic classification of lung cancer divides invasive adenocarcinoma of the lung into Lepidic, Acinar, Papillary, Micro papillary predominant and Solid predominant with mucin production [1]

PA is relatively rate and is more common in women and non-smokers. Radiological findings can vary from a solitary nodule, lung mass to lung infiltrates. PA has distinct pathological findings where in the alveolar architecture being replaced by papillae supported by fibro-vascular core.

Increasing knowledge of molecular abnormalities that drive human cancers offers the promise of therapies targeted at specific genetic lesions $[6,7]$. Genetic abnormalities may define a cancer at diagnosis and mutations may leads to acquired drug resistance. In May 2004, two studies showed that the presence of somatic mutations in the kinase domain of EGFR strongly correlates with increased responsiveness to EGFR tyrosine kinase inhibitors in patients with non-small cell lung cancer $[8,9]$.

Approximately $23 \%$ to $28 \%$ of patients with advanced nonsmall-cell lung cancer (NSCLC) have a high level of programmed death ligand 1 (PD-L1) expression (defined as at least $50 \%$ of tumor cells, regardless of the staining intensity). Studies indicated that patients with advanced NSCLC and a PDL1 tumor proportion score of $50 \%$ or greater were more likely than those with lower tumor proportion scores to have a response to Pembrolizumab, a highly selective, humanized monoclonal antibody against programmed death 1 (PD-1) that prevents PD-1 from engaging PD-L1 and PD-L2 [10].

The KRAS gene (Kristen rat sarcoma viral oncogene homolog) is located on chromosome $12 \mathrm{p} 12$. The gene is a member of the RAS oncogene family and encodes a GTPase. RAS proteins are involved in regulation of cell proliferation and cell survival pathways. Somatic missense KRAS are most commonly found in exons 2 and 3. KRAS mutations are detected in approximately $20-25 \%$ of non-small cell carcinoma of lung. Therapeutic targeting of KRAS-mutant lung adenocarcinoma represents a major goal of clinical oncology. Combinations targets for Trametinib, a MEK inhibitor and EGFR inhibitor combinations may lead to improve survival [11]. KRAS mutations may predict responsiveness to anti-PD-1/PD-L1 immunotherapeutic agents, however this remains under investigation.

Most patients with anaplastic lymphoma kinase (ALK)rearranged or ROS proto-oncogene 1 (ROS1)-rearranged nonsmall-cell lung cancer (NSCLC) are sensitive to tyrosine kinase inhibitor (TKI) therapy. Crizotinib showed marked antitumor activity in patients with advanced ROS1-rearranged NSCLC [12]. Lorlatinib, a novel, highly potent, selective, and brainpenetrant ALK and ROS1 TKI with preclinical activity against 
most known resistance mutations, in patients with advanced ALK-positive or ROS1-positive NSCLC $[13,14]$.

\section{Conclusion}

Over-all, papillary subtype has an intermediate prognosis, with some variability in survival rates among different studies, with a 5-year disease free survival of about $50-70 \%$ for Stage IIII. This case reiterates the importance of evaluating a nonresolving infiltrate with further diagnostic testing and tissues sampling. Adenocarcinoma of the lung can occasionally present as a lung infiltrate and delay and diagnosis can be detrimental in overall patient care.

\section{References}

1. Zugazagoitia J, Enguita AB, Nuñez JA, Iglesias L, Ponce S (2014) The new IASLC/ATS/ERS lung adenocarcinoma classification from a clinical perspective: current concepts and future prospects. J Thorac Dis 6: S526.

2. Silver SA, Askin FB (1997) True papillary carcinoma of the lung: a distinct clinicopathologic entity. Am J Surg Pathol 21: 43-51.

3. Brambilla E, Travis WD, Colby TV, Corrin B, Shimosato Y (2001) The new World Health Organization classification of lung tumours. Eur Respir J 18: 1059-1068.

4. Amin MB, Tamboli P, Merchant SH, Ordonez NG, Ro J, et al. (2002) Micropapillary component in lung adenocarcinoma: a distinctive histologic feature with possible prognostic significance. Am J Surg Pathol 26: 358-364.

5. Noguchi M, Morikawa A, Kawasaki M, Matsuno $Y$, Yamada T, et al. (1995) Small adenocarcinoma of the lung. Histologic characteristics and prognosis. Cancer 75: 2844-2852.

6. Greenman C, Stephens P, Smith R, Dalgliesh GL, Hunter C, et al. (2007) Patterns of somatic mutation in human cancer genomes. Nature 446: 153.
7. Papadopoulos N, Kinzler KW, Vogelstein B (2006) The role of companion diagnostics in the development and use of mutation-targeted cancer therapies. Nature Biotech 24: 985.

8. Lynch TJ, Bell DW, Sordella R, Gurubhagavatula S, Okimoto RA, et al. (2004) Activating mutations in the epidermal growth factor receptor underlying responsiveness of non-small-cell lung cancer to gefitinib. N Engl J Med 350: 2129-2139.

9. Paez JG, Jänne PA, Lee JC, Tracy S, Greulich H, et al. (2004) EGFR mutations in lung cancer: correlation with clinical response to gefitinib therapy. Science 304: 1497-1500.

10. Reck M, Rodríguez-Abreu D, Robinson AG, Hui R, Csőszi T, et al. (2016) Pembrolizumab versus chemotherapy for PD-L1-positive non-small-cell lung cancer. N Engl J Med 375: 1823-1833.

11. Manchado E, Weissmueller S, Morris JP, Chen CC, Wullenkord R, et al. (2016) A combinatorial strategy for treating KRAS-mutant lung cancer. Nature 534: 647.

12. Shaw AT, Ou SH, Bang YJ, Camidge DR, Solomon BJ, et al. (2014) Crizotinib in ROS1-rearranged non-small-cell lung cancer. N Engl J Med 371:1963-1971.

13. Shaw AT, Felip E, Bauer TM, Besse B, Navarro A, et al. (2017) Lorlatinib in non-small-cell lung cancer with ALK or ROS1 rearrangement: an international, multicentre, open-label, single-arm first-in-man phase 1 trial. Lancet Oncol 18: 1590-1599.

14. Russell PA, Wainer Z, Wright GM, Daniels M, Conron M, et al. (2011) Does lung adenocarcinoma subtype predict patient survival?: A clinicopathologic study based on the new International Association for the Study of Lung Cancer/American Thoracic Society/European Respiratory Society international multidisciplinary lung adenocarcinoma classification. J Thorac Oncol 6: 1496-1504. 